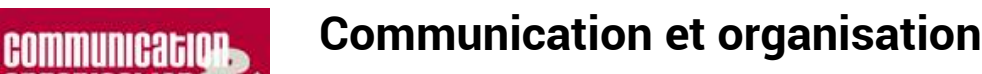

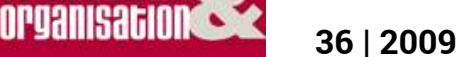

Pour une approche communicationnelle de l'individu au travail

\title{
Différences en signature entre la Chine et
} l'Occident

\section{Zheng Lihua}

\section{OpenEdition}

\section{Journals}

Édition électronique

URL : http://journals.openedition.org/communicationorganisation/1020

DOI : 10.4000/communicationorganisation. 1020

ISSN : 1775-3546

Éditeur

Presses universitaires de Bordeaux

Édition imprimée

Date de publication : 1 décembre 2009

Pagination : 238-249

ISBN : 978-2-86781-719-9

ISSN : $1168-5549$

Référence électronique

Zheng Lihua, « Différences en signature entre la Chine et l'Occident », Communication et organisation

[En ligne], 36 | 2009, mis en ligne le 11 mars 2011, consulté le 30 avril 2019. URL : http://

journals.openedition.org/communicationorganisation/1020 ; DOI : 10.4000/

communicationorganisation. 1020 
Analyses

\title{
Résumé
}

La signature est à la fois un signe d'identité de l'auteur et un signe de certification servant à valider un document. Comme signe d'identité, la signature révèle les traits identitaires de l'auteur et permet de l'identifier. Pourtant, les traits identitaires portés par la signature sont différents selon les cultures. En Occident, la signature révèle davantage les traits identitaires liés à la personne de l'auteur, par exemple, la présence physique lors de la signature, un esprit normal, une volonté libre, etc. En Chine, la signature révèle plutôt les traits identitaires liés à la position sociale de l'auteur, par exemple, la reconnaissance du groupe, les relations avec les autres, etc. Comme signe de certification, la signature a besoin de la reconnaissance des autres pour avoir la force de validation, ce qui est pareil en Chine et en Occident. Mais les critères de reconnaissance peuvent différer d'une culture à l'autre. En Occident, on insiste sur la constance de la signature et met en avant la personnalité indépendante de l'auteur. En Chine, c'est surtout la lisibilité de la signature qui prime, car il faut que les autres savent qui a signé. La lisibilité l'emporte sur la constance.

Mots-clés : Sociolinguistique, écriture, signature

\begin{abstract}
Signature is both a language symbol to express identity and a language behavior of certification. As an identity symbol, signature reflects the signer's characteristic, which helps to recognize who the signer is by reading his signature. However, different cultures have different attitudes on the characteristics shown by signature. In the west, signature is a reflection more of the individual characters of the signer, such as the physical presence, the mental normality, the free expression of will, and the preservation of identity, and so on. In china, however, signature is a reflection more of social characters of the signer, such as his social status and his relations with other people. The validity of signature, a language behavior of certification, relies on the social recognition, but different cultures have different standards of recognition. In the west, people emphasize the identicalness of signature, and the individuality of the signer, whereas in China, people emphasize the recognizability of signature, and the social recognition of the signer.
\end{abstract}

Key Words

Sociolinguistics, written language, signature

ZHENG Lihua est Professeur à Université des Etudes étrangères du Guangdong. 510420 - Guangzhou, Chine 


\section{Différences en signature entre la Chine et l'Occident

\author{
Zheng Lihua
}

zheng@mail.gdufs.edu.cn

« Signer » signifie, selon un dictionnaire chinois, « écrire de sa propre main son nom sur un document, une facture etc. pour en assumer la responsabilité » (Dictionnaire standard du chinois moderne, 2004). De cette définition, on peut au moins dégager quatre éléments : Premièrement, le mot signer est un signe linguistique doté d'une signification dénotative: en signant, le signataire se désigne. Deuxièmement, signer est une action. «Ecrire de sa propre main » implique que le signataire doit être présent en effectuant l'action. Troisièmement, signer n'est pas une action indépendante, car apposer son nom «sur un document, une facture, etc. » veut dire que cette action s'accomplit sur un support. Quatrièmement, «pour en assumer la responsabilité » nous montre que signer, au lieu d'être une action neutre, en est une sociale. Le signataire n'écrit pas simplement son nom, mais en le faisant, il valide un document et en devient responsable. Il s'ensuit que signer est à la fois un acte d'identification et de certification. Dans le « dictionnaire Hachette » (Hachette, 1998), nous trouvons une définition directe de la signature: "nom d'une personne, écrit de sa main sous une forme qui lui est particulière et constante, servant à affirmer la sincérité d'un écrit, l'authenticité d'un acte, d'une oeuvre, etc., à en assumer la responsabilité ». On peut remarquer que la définition française, entre autres choses, insiste sur la forme de la signature «qui lui est particulière et constante». Dans cet article, nous essayerons de montrer et d'analyser des différences en signature entre la Chine et l'Occident, à travers les données d'une enquête portant sur la certification ISO 9000 en Chine, que nous avons effectuée auprès des Chinois travaillant soit dans les entreprises chinoises, soit dans les entreprises à caractère multiculturel (entreprises à capitaux mixtes ou à capitaux étrangers).

\section{Le choix de la langue pour la signature}

Notre enquête nous montre que le choix de la langue des Chinois pour la signature dépend de deux facteurs : la situation et la personne. En général, dans les entreprises chinoises, en interne, on inscrit son nom chinois dans la signature: "Dans notre entreprise qui est une entreprise d'Etat, on ne reconnaît que le chinois dans la signature» (H., 20 ans, employé chargé de la certification ISO 9000). «Dans 
Analyses

notre Institut de Prévention contre le Tremblement de Terre, c'est le chinois qu'on emploie pour la signature » (H., 40 ans, responsable de la certification ISO 9000). Dans les entreprises à caractère multiculturel, on écrit souvent son nom étranger, parfois même pour un document en chinois, car dans l'entreprise, on se reconnait par le nom étranger lié étroitement à la personne portant ce nom : "Dans mon entreprise, qui est une entreprise française, j'utilise mon nom français 'Colette' pour la signature. Pour les documents chinois, je signe également 'Colette', car tout le monde sait que Colette, c'est $m o i »$ (F., 40 ans, responsable des projets). Si cette responsable des projets a l'habitude de mettre son nom français, c'est parce qu'elle travaille dans une entreprise française alors que dans les entreprises chinoises, c'est le chinois qui est la norme dans l'acte de signer. Tout cela nous démontre la force de la situation linguistique qui détermine le choix de la langue pour la signature. Une autre interviewée nous dit qu'en général, elle utilise son nom chinois pour la signature, mais que dans une situation informelle, elle peut aussi signer avec son nom étranger : "En contact avec les entreprises chinoises, j’utilise mon nom chinois. Dans une situation officieuse, où tout le monde est décontracté, je peux également mettre mon nom étranger dans la signature ». Cet exemple nous permet de voir, encore une fois, le rôle déterminant de la situation linguistique dans le choix de la langue concernant la signature, car dans une situation officielle, en contact avec les entreprises chinoises, elle doit recourir au chinois pour la signature.

Ce dernier exemple nous conduit au deuxième facteur déterminant le choix de la langue : le lecteur de la signature ou la personne à qui la signature est destinée. Certaines entreprises chinoises ont des relations commerciales avec des entreprises étrangères. Dans cette situation, on a recours au chinois pour signer des documents internes et à la langue étrangère pour des documents externes mais à deux langues pour les conventions ou les contrats : "Si c'est un document à usage interne, on emploie le chinois pour la signature car beaucoup d'employés chinois ne connaissent pas l'anglais. Pour les clients hongkongais, on signe des documents avec son nom anglais comme Peter, David, etc. mais s'il s'agit des contrats, il faut signer à la fois en chinois et en anglais. Sinon, on risque de ne pas être reconnu» (H., 33 ans, directeur); "Lorsque je signe les contrats avec les entreprises étrangères, j'utilise le chinois pour l'exemplaire en chinois et le 
pinyin ${ }^{190}$ pour l'exemplaire en langue étrangère » (H., 33 ans, directeur). De même, les Chinois qui travaillent dans les entreprises étrangères utilisent la langue étrangère à l'interne mais en contact avec les clients chinois, ils doivent signer en chinois : «En général, dans mon entreprise qui est une entreprise française, je signe 'Polo', mais avec les usines chinoises, je dois signer en chinois. Par exemple, je leur passe des bons de commande, des documents pour la fabrication, c'est mon nom chinois que je mets, car dans les usines chinoises, on a très peu de contact avec la langue étrangère » (H., 25 ans, employé).

Il vaut la peine de nous demander d'emblée pourquoi il faut signer en chinois avec les Chinois. À cette question, les interviewés ont donné deux réponses. Premièrement, il y a le problème de la reconnaissance. On risque de ne pas être reconnu si l'on signe en langue étrangère avec les Chinois : "Bien que ce soit plus simple et plus rapide lorsque je signe en français ou en anglais, cela pourra poser problème dans les usines chinoises, car les Chinois, qui ne connaissent pas la langue étrangère, peuvent douter de l'authenticité de votre signature et se demander si c'est vraiment votre nom » (F., 40 ans, responsable des projets). Deuxièmement, c'est un signe de respect. Utiliser le chinois pour les entreprises chinoises, c'est se montrer poli à leur égard : «Je mène une négociation avec des clients chinois. Après, il faut signer les protocoles. Pour être polie, je signe en chinois» (F., 40 ans, responsable des projets). «Si vous mettez votre nom étranger dans la signature avec les Chinois, ils vont penser que vous êtes en train de faire parade » (H., 33 ans, directeur des projets).

Nous voyons par là que la signature est une communication situationnelle, dans laquelle, le facteur-situation joue un rôle primordial. Dans les entreprises chinoises, on a recours au chinois pour la signature alors que dans les entreprises à caractère multiculturel, c'est la langue étrangère qui est en usage. Une signature en langue étrangère paraîtra bizarre dans un contexte uniquement chinois. Un autre facteur pertinent auquel dépend le choix de la langue pour la signature, c'est la personne à qui la signature est destinée. On peut signer en langue étrangère avec les étrangers ou avec les Chinois habitués à l'usage de la langue étrangère comme les hongkongais, mais avec les Chinois et surtout avec les Chinois qui ne connaissent pas la langue étrangère, c'est le chinois qui doit être utilisé dans la signature. Ces deux facteurs (la situation et la personne), déterminants

${ }^{190}$ Le pinyin est un système de transcription phonétique des caractères chinois en alphabet. 
pour la signature, constituent des normes implicites régissant l'acte de signer. Si l'on ne respecte pas ces normes, on risquera de ne pas être reconnu comme acteur social ou de paraître impoli à l'égard des lecteurs de la signature.

Un phénomène lié au choix de la langue pour la signature mérite notre attention. Il s'agit du «bilinguisme» dans la signature. Pour bien illustrer ce point, prenons l'exemple d'une interviewée, responsable des projets dans une entreprise française : Son nom chinois est 李雅, et comme beaucoup de Chinois qui travaillent dans les entreprises étrangères, elle a adopté un nom français «Colette». Donc, pour la signature, elle dispose de trois choix : son nom chinois en caractères

李雅 ou le pinyin de ce nom « Li $\mathrm{Ya} »$ ou son nom français «Colette». Du point de vue des formes d'écriture, il s'agit de trois signes visiblement très différents, mais dans son travail, elle les considère tous comme son nom, seulement en les utilisant dans les contextes différents et avec des personnes différentes. Ce phénomène ressemble au bilinguisme dans une communication orale et nous pouvons le nommer «bilinguisme»dans la signature. Ce dernier bilinguisme est défini par rapport au premier. Le bilinguisme en oral désigne «la qualité d'une personne, d'une population qui parle deux langues» (Dictionnaire Hachette: 203). En fait, le terme de bilinguisme est applicable à trois, quatre, cinq langues ou plus (MACKEY, 1997 : 61). Par exemple, une personne peut parler le chinois, le français et l'anglais. Le bilinguisme dans la signature fait référence au fait qu'on a deux ou plus de deux formes graphiques disponibles pour la signature. Par exemple, les trois formes de signature d'une même personne : 李雅, « Li Ya » et «Colette». Cela veut dire qu'une même personne possède trois moyens pour sa signature et que pour elle, c'est toujours sa signature, c'est-à-dire sans distinction sur le plan de la dénotation. Pourtant, nous nous demandons quelles sont les raisons sociolinguistiques qui sous-tendent le choix des possibilités, c'est-à-dire pourquoi dans certains contextes, c'est une des trois possibilités choisie plutôt qu'une autre. En effet, le «bilinguisme» dans la signature ne tombe pas du ciel. Pour apparaître, il faut y avoir au moins deux conditions, une externe et l'autre interne. La condition externe est fournie lorsque deux écritures sont en contact comme deux langues se rencontrent dans la communication orale. Par exemple, pour signer un contrat chinois en deux exemplaires, on utilise les caractères chinois comme 李雅 et on n'a même pas l'idée d'utiliser le pinyin « $\mathrm{Li} \mathrm{Ya} »$ ou le français 
comme «Colette». Le choix entre les trois formes graphiques est possible lorsqu'il y a le contact entre les écritures. Par exemple, lorsque le contrat a deux exemplaires, l'un en chinois et l'autre en français, le signataire peut choisir entre le chinois, le pinyin ou le français. La condition interne désigne la capacité des personnes pour le choix des formes graphiques tout comme dans une communication orale, on a la capacité de parler des langues différentes. Par exemple, devant les exemplaires en chinois et en français d'un même contrat, le Français ne peut que signer son nom français, car en général, il n'a pas la capacité de signer en chinois. Mais un Chinois, normalement, dispose au moins de deux possibilités, les caractères de son nom chinois (comme 李雅 et le pinyin de ce nom (comme «Li Ya»), car le chinois lui-même a deux formes d'écriture, l'une en caractères qui note les formes graphiques et l'autre en pinyin qui transcrit la phonétique et qui ressemble en forme à une langue occidentale. Donc, en théorie, pour signer un document en langue étrangère, un Chinois a deux possibilités: les caractères chinois et le pinyin. Pour les personnes travaillant dans les entreprises étrangères, elles ont une troisième possibilité avec leur nom étranger. En général, comme notre enquête le montre, les Chinois utilisent les caractères pour signer un document en chinois, mais devant un document en langue étrangère, les Chinois, bien qu'ils disposent de trois possibilités (les caractères chinois, le pinyin et la langue étrangère), recourent le plus souvent au pinyin ou à la langue étrangère. En fait, ce qui détermine réellement le choix de la langue, c'est moins la langue utilisée par le document que la personne cachée derrière le document: avec les Chinois, il faut signer en chinois et avec les étrangers, il faut signer en langue étrangère. C'est la raison pour laquelle les interviewés chinois, lorsqu'ils décrivent le choix de la langue pour la signature, utilisent des mots comme « reconnaissance », « douter», « respect », « poli », «faire parade», termes plus ou moins liés au jugement dans les relations interpersonnelles.

Les analyses ci-dessus nous montrent que chez les Chinois, la situation et la personne sont deux facteurs-clé dont dépend le choix de la langue pour la signature. Pour les Chinois, la signature n'est pas seulement un signe d'identité mais aussi une marque de reconnaissance. Si la signature était un simple signe d'identité, le signataire serait libre dans son choix, c'est-à-dire qu'il peut signer avec les caractères de son nom chinois ou le pinyin de ce nom ou son nom étranger, à condition qu'il reconnaisse lui-même que le signe qu'il a choisi est le symbole unique de son identité. Mais une signature chinoise a besoin d'être approuvée par le groupe. Si tout le 
Analyses

monde signe avec les caractères chinois, vous ne pouvez pas utiliser le pinyin ou le nom étranger. Si le groupe n'arrive pas à déchiffrer votre signature ou refuse de le faire, la signature perdra sa valeur en tant que signe.

\section{La constance et la non constance de la signature}

Le choix de la langue pour la signature est déjà un exemple montrant que la signature peut ne pas être constante dans le contexte chinois. Ici, ce qui nous préoccupe, c'est la non-constance à l'intérieur d'une même langue. En Chine, lorsqu' on écrit les caractères chinois, ceux-ci peuvent varier en style ou en régularité et représentent parfois des formes bien différentes. Dans notre enquête, les interviewés proposent plusieurs explications à la non-constance de la signature. D'abord, le temps : "la signature peut évoluer. Avec le temps, elle peut devenir de plus en plus illisible ou de plus en plus lisible» $(\mathrm{H}, 53$ ans, directeur d'usine). Puis, le contexte. Si l'on signe une fois beaucoup de documents, on ne peut pas maintenir l'uniformité de la signature : "Parfois, j'ai à signer des centaines de dessins. Au début, j'écris avec soin, mais à la fin, je ne peux plus et la forme peut changer» (H., 23 ans, employé). Enfin, la personne : "Lorsque je signe un document soumis par l'échelon inférieur, je le fais en un seul trait. Mais si c'est quelque chose pour le directeur général, je dois écrire lentement et avec beaucoup de soins» (H., 52 ans, directeur des projets). Nous voyons par là que la constance ne semble pas un critère à respecter strictement pour une signature chinoise.

Quand les Chinois décrivent la variation de la signature, ils semblent admettre implicitement la non-constance de celle-ci. Pour eux, la signature peut varier avec le temps et en fonction de la tâche et de la personne liée à la signature. Une signature bien soignée est le reflet d'une attitude sérieuse ou la marque de respect. Ce qui est pertinent dans une signature chinoise, ce n'est pas son uniformité, mais son caractère social, étant donné que si c'était la constance qui prime, il n'y aurait pas de différences entre une signature bien soignée et une signature mal tracée, car une signature bien soignée peut être constante, mais une signature mal tracée pourra l'être aussi. Mais si c'est le caractère social de la signature qui est déterminant, une signature bien soignée ou mal tracée transmet un sens, car elle reflète l'attitude de l'auteur à l'égard de son lecteur et les différentes formes graphiques acquièrent ainsi des significations sociales différentes.

En Occident, la constance de la signature constitue un principe fondamental. Cette constance représente celle du signataire. D'abord, une signature constante signifie que le signataire possède une capacité 
constante lui permettant d'assumer la responsabilité liée à la signature. La constance de la signature n'est pas comme celle du tampon avec lequel, on peut avoir deux empreintes qui se superposent complètement grâce à la matrice commune. Mais deux signatures tracées à la main ne peuvent pas s'ajuster tout à fait. Cela veut dire que la constance de la signature est relative et qu'elle est maintenue non pas mécaniquement, mais par le signataire qui doit bien mettre en coordination ses yeux, sa main et son corps. Donc, par la constance de la signature, le signataire nous démontre qu'il maîtrise bien son corps et qu'il maintient la volonté et la capacité d'assumer la responsabilité (FRAENKEL, 1995 : 74). Puis, la constance de la signature signifie également la constance de la promesse. La signature est en fait une promesse écrite, une responsabilité à l'égard d'un projet à accomplir dans le futur. Le temps peut ainsi mettre en doute cette responsabilité lointaine. Par une signature constante, le signataire nous témoigne que sa promesse ne changera pas bien que le monde peut changer ou que sa vie peut être différente. Enfin, dans la signature, le signataire utilise son propre nom, cela veut dire que le garant du signataire est son propre nom. Et écrire toujours de la même manière son nom dans la signature signifie que le signataire reste fidèle à son propre nom et à la promesse porté par ce nom.

\section{La lisibilité et l'illisibilité de la signature}

La lisibilité de la signature est un critère important pour les Chinois. D'abord, ils pensent que c'est une responsabilité d'être reconnu comme l'auteur de la signature: "Les autres reconnaissent ma signature. Il faut qu'ils la reconnaissent. Signer est en fait une action sacrée. Il faut en prendre la responsabilité. Une signature lisible pour les autres est fondamentale» (H., 53 ans, responsable de la production). Puis, pour les Chinois, si la signature n'est pas lisible, les autres peuvent ne pas exécuter ce qui est écrit dans le document et la signature perdra automatiquement sa valeur : "Si on ne reconnaît pas votre signature, on peut ne pas appliquer le document, car on peut dire qu'on ne connaît pas la personne qui a signé » (H., 53 ans, responsable de la production). Donc, la signature peut être mal tracée, mais il faut qu'elle soit lisible: "Par exemple, quand j'écris mon nom 陈, je le trace rapidement. On peut dire que c'est joliment écrit ou non, mais au moins, il faut que les autres reconnaissent que c'est le mot 陈 que j'écris» (H., 33 ans, directeur des projets). Les interviewés ont bien noté la différence entre une signature chinoise et une occidentale : "Si on signe avec un nom anglais, la signature ne 
Analyses

doit pas être reconnue. Si elle est reconnue, il ne faut pas qu'elle soit imitable » (H., 33 ans, directeur des projets).

On voit par là que la lisibilité est considérée par les Chinois comme un principe fondamental. Cette signature peut être tracée rapidement (une écriture cursive), mais il faut qu'elle soit lisible, c'est-à-dire que les autres reconnaissent la personne qui a signé. $\mathrm{Si}$ une signature bien tracée ou mal tracée reflète l'attitude de l'auteur à l'égard du document ou de la personne lisant le document, la lisibilité de la signature relève de la moralité de l'auteur. Par ailleurs, une signature illisible perdra sa valeur en tant que signe. Une anecdote concernant la signature est bien révélatrice. Peu après la fondation de la nouvelle Chine, dans les années 1950, ZHOU Enlai, premier ministre chinois, avait chaque jour beaucoup de documents à signer. Pour être plus rapide, ZHOU, en adoptant le style cursif, a simplifié son nom 周 et l'a tracé comme un triangle. Après, il a reçu une lettre venant d'un vieux membre du parti le critiquant directement: "Monsieur le premier ministre, les documents de l'Etat que vous avez signés sont à être appliqués dans tout le pays. Mais lorsque nous recevons les documents, nous ne pouvons pas reconnaître votre signature. Comment pouvons-nous les appliquer ? » Après avoir reçu cette lettre, ZHOU a compris que les dirigeants de divers échelons, à cause de la révolution, n'avaient pas eu une formation suffisante et qu'un mot mal tracé risquait de ne pas être reconnu et de poser problème au travail. ZHOU a bien accepté la critique et a signé des documents avec son nom tracé lentement et avec soin pour qu'il soit complètement lisible (JIN, 1999 : 15). De cette anecdote, nous pouvons remarquer qu'aussi bien pour ZHOU que pour la population, la signature doit être lisible. Une signature illisible transgresse la norme.

Notre enquête a révélé une différence entre la signature chinoise et la signature occidentale. Selon nos interviewés chinois, « une signature lisible pour les autres est fondamentale », mais «si on signe avec un nom anglais, la signature ne doit pas être reconnue ». En effet, nous constatons que les signatures occidentales sont, contrairement à celles chinoises, illisibles. En lisant la signature, on peut rarement reconnaître l'auteur. Du point de vue sémiologique, une signature possède essentiellement deux caractéristiques : celle liée au caractère autocentrique et celle liée à la marque d'identification (HARRIS : 1993 : 205). Une signature occidentale nous semble d'abord être la marque d'un individu indépendant des autres, car on insiste sur son caractère autocentrique qui pousse le scripteur à chercher une configuration unique et personnelle et à inventer effectivement son 
propre signe écrit en toute liberté. Ce trait est même considéré comme le symbole de la liberté : «Dans l'histoire de la civilisation occidentale, le développement de la signature en tant que signe et le développement des droits de l'individu, tant politiques qu'économiques, s'enchevêtrent d'une façon tout à fait remarquable » (HARRIS, 1993 : 207). C'est ce qui fait que, souvent, les signatures occidentales sont illisibles pour les autres. Et pour compenser cette illisibilité, le signataire met au-dessus ou au-dessous de sa signature, son nom imprimé en toutes lettres ou écrit en majuscules, expédient qui ne laisse aucun doute au sujet de l'identité du signataire. Une signature chinoise est avant tout la marque d'une personne faisant partie du groupe et on insiste sur son caractère de marque d'identification. Elle doit être reconnaissable et lisible par les autres. La règle de lisibilité prime sur celle de l'originalité.

\section{Conclusion}

Dans cet article, nous avons parlé du choix de la langue pour la signature, de la constance et de la lisibilité de celle-ci. Ces trois problèmes concernent la lecture de la signature. En effet, la signature est fondamentalement un signe écrit. Et comme tout signe écrit, elle est destinée à être lue, ce qui constitue le trait social de l'écriture. Tout signe écrit perdra de sens s'il n'est pas écrit pour être lu. Le problème, c'est que dans les différentes cultures, ce caractère social peut se manifester différemment. Les Chinois placent au premier rang la lisibilité de la signature et insistent sur la reconnaissance du groupe à l'égard de la signature. Les Occidentaux privilégient la constance de la signature et la considèrent comme représentative de la personne et de ses comportements. En Chine, on peut signer en plusieurs langues, écrire son nom lentement ou le tracer rapidement, à condition que les autres puissent reconnaître l'auteur de la signature. En Occident, bien qu'une signature soit correctement tracée et clairement lisible pour les autres, elle n'aura pas de valeur si elle ne correspond pas à la signature préalablement posée par l'auteur. Par exemple, dans une banque française, lorsqu'on veut faire un retrait, la banque exige qu'on présente, à part sa pièce d'identité, une signature correspondant à celle déposée à la banque. On peut écrire son nom très lisiblement et très soigneusement, la banque peut très bien refuser de l'accepter comme signature, s'il ne correspond pas du tout à la signature habituelle.

La signature est à la fois un signe d'identité de l'auteur et un signe de certification servant à valider un document. Comme signe d'identité, la signature révèle les traits identitaires de l'auteur et permet de l'identifier. Pourtant, les traits identitaires portés par la signature sont 
différents selon les cultures. En Occident, elle révèle davantage les traits identitaires liés à la personne de l'auteur, par exemple, la présence physique lors de la signature, un esprit normal, une volonté libre, etc. En Chine, la signature révèle plutôt les traits identitaires liés à la position sociale de l'auteur, par exemple, la reconnaissance du groupe, les relations avec les autres, etc. Comme signe de certification, la signature a besoin de la reconnaissance des autres pour avoir la force de validation, ce qui est pareil en Chine et en Occident. Mais les critères de reconnaissance peuvent différer d'une culture à l'autre. En Occident, on insiste sur la constance de la signature et on met en avant la personnalité indépendante de l'auteur. En Chine, c'est surtout la lisibilité de la signature qui prime, car il faut que les autres sachent qui a signé. La lisibilité l'emporte sur la constance.

L'importance accordée à la personnalité indépendante ou au groupe peut être révélée par une comparaison entre la signature et le tampon, les deux servant à identifier l'origine de l'écriture et à la valider dans sa fonction de preuve. C'est ici qu'émergent un certain nombre de différences entre les deux cultures. Si beaucoup d'entre elles recourent à la signature personnelle ou au tampon d'une collectivité pour valider un document écrit, il semble que la valeur qu'elles y accordent diffère. En Chine, le tampon, qui a derrière lui la caution d'une collectivité, pèse beaucoup plus lourd que la signature, alors qu'en Occident, la signature qui implique la responsabilité personnelle du signataire visà-vis de sa signature semble l'emporter sur le tampon qui ne définit pas clairement les personnes responsables. Si en Chine, un seul tampon rouge peut suffire et qu'en Occident, une seule signature peut suffire, l'inverse ne semble pas vrai lorsqu' on change de pays.

Les différences en signature entre la Chine et l'Occident sont en fait le reflet des différences culturelles. Si dans toute société, l'individu est inévitablement membre d'une communauté donnée, le sens communautaire revêt une dimension particulièrement importante en Chine. Dans la culture chinoise, chaque individu a sa place dans le réseau qu'il forme avec un autre ou des autres. Cette place est classificatoire : il est fils par rapport à ses parents, mari par rapport à sa femme, père par rapport à ses enfants, et ainsi de suite. Chaque statut comporte des devoirs spécifiques. Ces différents statuts sont cumulatifs de même que les devoirs qui leur incombent. L'individu dépend d'autrui pour réaliser sa valeur sociale car elle est définie par le groupe et non pas par lui-même, tout comme son image elle-même est contrôlée par l'opinion du groupe. Le confucianisme définit ainsi l'homme social par le mot ren (仁) dont la structure est ici 
révélatrice : «homme + deux », c'est-à-dire qu'on ne peut définir un homme que par rapport à un autre homme. D'ailleurs, ce sont les autres (le groupe, la société, etc.) qui décident qu'un homme est « bon» ou « mauvais ». En effet, en chinois, l'expression 人品» (ren pin) signifie «qualité, personnalité de l'homme». Le graphisme du terme 品》(pin) est composé de trois bouches. La qualité d'un homme est donc déterminée par la parole des autres (ZHENG, 1995 : 60). Ainsi, la régulation entre les individus est réalisée par le contrôle du groupe, et les comportements d'un individu sont sanctionnés par le regard ou le dire des autres. La culture chinoise, fondée sur le groupe, insiste sur le collectivisme, à l'encontre de la culture occidentale qui, structurée sur les individus, privilégie l'individualisme (CHEN, 2004 : $320)$.

\section{Bibliographie}

CHEN(X. M.), Les voyageurs et les étrangers, Beijing: Sciences en éducation, 2004.

JIN (Y.), Le changement de la signature du premier ministre ZHOU Entai, in Les activités du bureau, 1999(6).

HARRIS (R.), La sémiologie de l'écriture, Paris: CNRS Editions, 1993.

FRAENKEL (B.), La traçabilité d'une fonction caractéristique des écrits de travail. In : Connexion $n^{\circ}$ 65, 1995.

MACKEY(W.F.), Bilinguisme, In: Marie-Louise Moreau (éd.), Sociolinguistique, concepts de base, Paris, Mardaga, 1997.

ZHENG (L.H.), Les Chinois de Paris et leurs jeux de face, Paris : L'Harmattan, 1995.

Dictionnaire standard $d u$ chinois moderne, Beijing: Editions des langues étrangères et éditions du chinois, 2004.

Dictionnaire Hachette encyclopédique illustré, Paris : Hachette, 1998. 